\title{
Roles for Personal Informatics in Chronic Pain
}

\author{
Sergio Felipe ${ }^{1}$, Aneesha Singh ${ }^{1}$, Caroline Bradley, Amanda CdeC Williams, Nadia Bianchi-Berthouze \\ UCLIC, University College London, London, UK
}

\begin{abstract}
Self-management of chronic pain is a complex and demanding activity. Multidisciplinary pain management programs are designed to provide patients with the skills to improve, maintain functioning and self-manage their pain but gains diminish in the long-term due to lack of support from clinicians. Sensing technology can be a cost-effective way to extend support for self-management outside clinical settings but they are currently under-explored. In this paper, we report studies carried out to investigate how Personal Informatics Systems (PIS) based on wearable body sensing technology could facilitate pain self-management and functioning. Five roles for PIS emerged from a qualitative study with people with chronic pain and physiotherapists: (i) assessment, planning and prevention (ii) a direct supervisory and co-management role, (iii) facilitating deeper understanding, (iv) managing emotional states, and (v) sharing for social acceptability. A web-based survey was conducted to understand the parameters that should be tracked to support self-management and what tracked information should be shared with others. Finally, we suggest an extension to previous PIS models and propose design implications to address immediate, short-term and longterm information needs for personal use of people with chronic pain and for sharing with others.
\end{abstract}

Keywords-Chronic pain, quantified-self, self-management, physiological sensing, emotional wellbeing

\section{I.INTRODUCTION}

Grouped under the umbrella term of Personal Informatics Systems (PIS) [1], self-tracking technologies are defined by the activities of collecting, visualising and reflecting on personal information to increase selfawareness, facilitate behaviour change and promote wellbeing [2]. Barriers to access evidence-based treatments for long-term conditions such as diabetes, heart disease or chronic pain (CP) have made interventions using sensing technology promising because of their potential for improving clinical outcomes [3]. In our study, we are interested in understanding the opportunities that such technology can offer to people with musculoskeletal CP where physical activity is limited by both physical and psychological factors.

Most technologies aimed at self-management of chronic conditions have focused on addressing behavioural and lifestyle factors (e.g. diet, physical exercise) or the recovery of movement (e.g. in stroke) [3]. Although these are common problems to most long-term conditions, people with CP are also confronted with unique psychological barriers such as pain-related fear and anxiety when approaching physical activities [4]; these barriers can result in fear-avoidance behaviours and withdrawal from daily activities leading to increased disability and depression [5]. Also, pain demands attention [6] and the planning of daily activities to optimize the use of limited physical and psychological resources is central to daily life of people with

${ }^{1}$ First authorship is shared by the first two authors.
CP [6]. Moreover, given that CP is invisible, i.e. no physical evidence might be present to justify the persistent pain, people with $\mathrm{CP}$ find it difficult to understand and accept their condition and may also feel misunderstood by others [7] [8].

The qualitative studies presented by Singh et al. [9], uncovered needs and barriers of people with $\mathrm{CP}$ in remaining physically active and identified opportunities for sensing technology to help people with $\mathrm{CP}$ to maintain a program of physical activity. They implemented and extended strategies used by physiotherapists and people with $\mathrm{CP}$ through the use of aural feedback to support exercise sessions [9]. In this paper we extend that work by investigating how PIS could help people with CP to better understand their condition and how personalised guidance provided by PIS could help them to improve selfmanagement practices in everyday functioning beyond physical activity sessions. For this, we conducted a focus group with pain specialist physiotherapists and interviews with people with CP. A web-based survey was used to gather further details about what information people with CP would like to track to facilitate self-management and who they would share this information with. We conclude the paper by discussing the themes that emerged from these studies and how wearable sensing technology could support $\mathrm{CP}$ self-management. We also suggest the need for extending previous PIS models based on our findings.

\section{BACKGROUND}

\section{A. Chronic Pain and its management}

Chronic (persistent) pain (CP) is defined as pain that lasts for more than twelve weeks, or when pain persists beyond the time of healing [10]. CP is due to changes in the central and peripheral nervous system resulting in amplification of pain signals [6]. Despite absence of tissue or bone damage, people with CP may still interpret pain as a sign of serious bodily harm and due to fear of increasing pain or causing further damage [5], they may adopt protective behaviours and avoid certain movements. Such avoidance behaviours may be reinforced due to temporary increases in pain; for example, because the person may try movements $\mathrm{s} / \mathrm{he}$ is unused to or generally avoids. This can lead to a cycle of progressively limiting the amount of physical activity and participation in daily life [5].

Besides fear and avoidance, catastrophising thoughts exaggerate and magnify the threat posed by pain making it difficult to shift the focus away from pain [11]. Catastrophising thoughts can also influence the transition from acute to $\mathrm{CP}$ [5]. Feelings of anxiety and of hopelessness about the CP condition are additional barriers to resuming normal activity, and can discourage people from pursuing valued activities [4].

Because of the effects of CP on all aspects of an individuals' wellbeing, a multifaceted methodology for the 
treatment of pain has been formulated taking into account social, economic, physical and cognitive aspects of the $\mathrm{CP}$ experience. Cognitive-behavioural techniques (CBT) are used to change the person's beliefs about pain and to facilitate the adoption of adaptive coping strategies [12]. Among these are increasing awareness through selfobservation, belief reappraisal, and affective regulation [13], that patients can use to identify and challenge unhelpful beliefs behind maladaptive behaviours and catastrophising thoughts [14]. An important skill is the pacing of activities by distributing them across the day or modifying activities (e.g. taking breaks) to confront maladaptive cycles [13]. People with $\mathrm{CP}$ also use relaxation techniques to facilitate movement-based activity, counterbalance physical tension or to increase the positive effects of taking rest breaks [15].

A critical goal of pain management programs is for patients to use these skills on their own in everyday functioning, which can be difficult, since they might feel less safe when away from the support of specialists [15]. Singh et al. [9] identified strategies used by physiotherapists and people with CP in overcoming barriers the latter face during physical activity. They suggested different strategies employed by people according to their stage in the pain management journey: explore and understand one's body capabilities, focus on pleasurable experience produced by one's body, listen to one's body cues to normalize them, take responsibility for pacing activity, and reduce exposure to negative emotions [9]. Gromala et al. [7] identified barriers that people with $\mathrm{CP}$ encounter in maintaining a social life, including how they carefully manage their social interactions to maximise chances of success or withdraw in case of disruptions or unexpected events. Both these studies [7][9] suggest the use of technology to support and enhance personal strategies used by people with $\mathrm{CP}$ to overcome pain-related barriers.

\section{B. Technology and chronic pain}

There is some evidence that technology-based interventions based on multidisciplinary approaches can increase functional activity and self-efficacy [16], and reduce pain severity, pain-related emotional distress and fear, catastrophising and perceived disability [13][17]. These interventions may include features for helping patients with exercise and stretching, relaxation, goal-setting, or cognitive restructuring (CBT) [18]. One of their core components is the self-collection of pain-related information from questionnaires and pain diaries [19]. This data can be shared with practitioners to provide a rich picture for assessment and monitoring, enhance patient-carer communication and facilitate prevention of setbacks [20]. For example, in a study with a group of 120 women who self-reported mood and activities, and received personalised CBT-based feedback from a therapist, the authors reported reduced catastrophising [17]. Moreover, by collecting and reflecting on this information patients can become more aware of the characteristics of their pain (e.g. intensity, location, triggers) [18], and more engaged in self-managing activity [21]. For instance, web-based tools allow people to report on pain episodes or levels of activity at the end of the day, and can be used by patients at their own pace and time [19]. Smartphone apps developed for CP allow people to report events as they happen [22], and to record parameters such as pain location and intensity more intuitively using touchenabled interfaces (e.g., 3D rotating human figure, sliders for pain intensity) [23]. Apps provide functionality such as examples of training exercises and relaxation techniques (e.g., Pocket Therapy, www.scavomed.com), or feature selfreported pain-tracking (e.g. Chronic Pain Tracker, www.chronicpaintracker.com), setting goals and receiving messages of support (e.g., WebMDPainCoach, www.webmd.com/webmdpaincoachapp). But, as pointed out by [24] adherence rates for these applications are often low due to time and effort required to enter self-reported data instead of automatic tracking of objective information through sensing devices [25]. In addition, they do not support everyday functioning, address psychological barriers of CP or use the identified strategies [9] [7].

\section{Body sensing-Technology to facilitate physical activity}

A recent wave of commercial products are using the sensors integrated into wearable devices [26] to infer activities and physiological states. These are combined with smartphone apps and web dashboards for reviewing progress based on fitness (Nike+), wellness goals (Jawbone UP, Fitbit Flex), or more specific tracking such as correcting posture (LUMOback). Other activities beyond walking and running can now be recognised. The RecoFit system uses an armworn inertial sensor to automatically monitor repetitive exercises (e.g. weight training), discriminate between exercise and non-exercise periods and count exercise repetitions [27]. Besides facilitating and promoting physical exercise, sensors have been used for other clinical purposes, e.g. to support physical rehabilitation to initiate movement [28] and correct movement [29].

However, for $\mathrm{CP}$, simply sensing activity is not sufficient and psychological aspects and fluctuation of pain levels need to be taken into account. Gromala et al. [30] investigated the use of virtual reality to provide a digital environment to facilitate relaxation while walking in a virtual space. Singh et al. [9], explored how movement sensing technology could increase confidence and selfefficacy in physical activity. They proposed using structured personalized sonification representing the tracked movement while the person engaged in rehabilitation exercises. The results showed that people with $\mathrm{CP}$ felt more confident of performing feared movements and able to engage with the movement, as the sound provided reassurance by continuously informing them of their body configuration. In addition, because these devices can be worn during everyday activities, they offer new opportunities to capture and understand the $\mathrm{CP}$ experience outside of clinical settings.

These studies provide examples of using sensing technology to support people with $\mathrm{CP}$ in physical activity sessions, not everyday functioning. Our study aims to gather an understanding of the aspects of PIS that are important for everyday functioning, and to propose avenues for PIS design addressing aspects specific to the $\mathrm{CP}$ condition.

\section{METHOD}

We conducted qualitative studies to investigate how body-sensing PIS technology could support selfmanagement in musculoskeletal $\mathrm{CP}$ with a focus on facilitating maintenance of an active life. In particular, we were interested in gaining an understanding of (i) the types of functionality that would be useful (i.e., what should be tracked), (ii) the reasons why these would be beneficial, and (iii) who should have access to this information. Three consecutive studies were conducted: 
- A focus group (1-hour) with three CBT physiotherapists (10-18 years of experience) from the Pain Management Centre, University College London Hospitals.

- Semi-structured interviews (25 to $65 \mathrm{~min}$, average length $42 \mathrm{~min}$ ) with nine people (5 women, 4 men, aged 25 - 67 years) with musculoskeletal CP (between 6 - 28 years), enlisted from local pain groups or from previous studies.

- A web-based survey with 33 questions (available at www.emo-pain.ac.uk/PISsurvey) filled by 35 people with $\mathrm{CP}$ (26 female and 9 male, average age $=51$, std $=13$ ). All participants had CP for more than 1 year.

We interviewed physiotherapists for their expertise on what is beneficial for people with $\mathrm{CP}$ when self-managing physical activity. People with $\mathrm{CP}$ were interviewed to understand their needs and how PIS could address the needs.

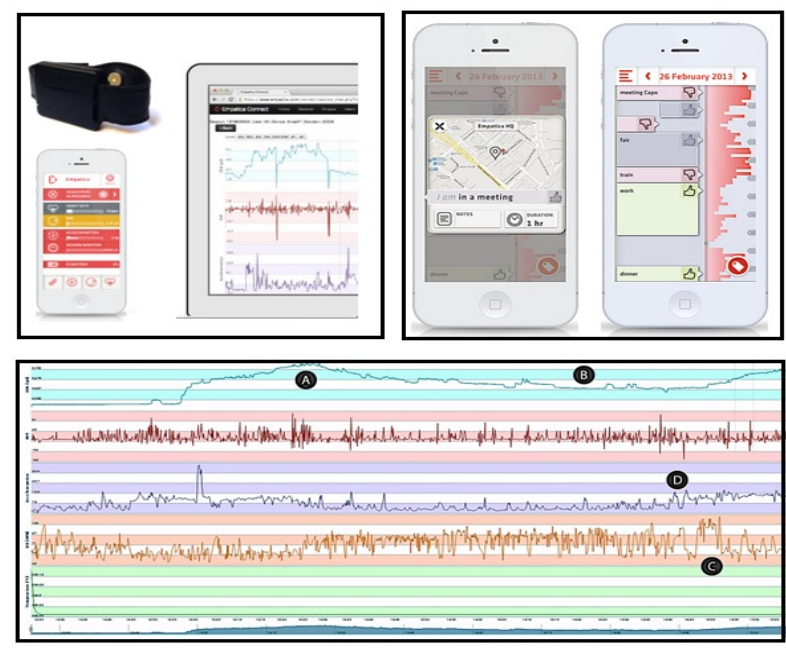

Fig. 1. Top left: Empatica device, app main menu and dashboard. Top right: Location detection and emotional state visualizations. Bottom: Biometric data from Empatica's online dashboard, showing a 3-hour session collected to demonstrate PIS data reviewing aspect. The graph showed higher stress levels during working hours (A), which decreased when the person walked to an after-work social gathering (B). Also, heart rate increase $(\mathrm{C})$, and motion sensor data when brisk walking (D).

\section{A. Materials}

Since PIS are relatively novel (especially at the time of the study: interviews conducted in August 2013, webbased survey conducted in early 2015), we provided an overview of these systems and their possibilities. This helped to facilitate discussion of how they could be useful with participants' own pain management strategies. The Empatica $^{2}$ bio-sensing device (www.empatica.com) was chosen for its ability to demonstrate the main uses identified through our literature review: activity and biosensing tracking, and rich presentation and contextualization of tracked data. The Empatica system (Fig. 1 - top left) measures movement and activity using an accelerometer and gyroscope embedded on a bracelet, and tracks location using GPS mapping. The bracelet sensors also track biomarkers such as heart rate, temperature and galvanic skin response (GSR), which provide real-time feedback to a smartphone app where users can also log notes. A "stress-o-meter" function on the app can infer the user's level of stress or relaxation in real-time from collected biomarkers. This data

\footnotetext{
2 This study was not supported by Empatica
}

can be uploaded to a web visualisation dashboard, allowing users to review sessions retrospectively. Data can also be linked through a geographical map to the place the person was at the time of tracking and comments can be entered on the map.

For the web-based survey, two more PIS applications were listed together with their URL for the participants to visit: (i) Fitbit for activity and sleep tracking (e.g., $w w w$.fitbit.com/uk), and (ii) Apps for smartphones (e.g., www.azumio.com/s/instantheartrate/index.html). The LimeService was used for hosting the survey (www.limeservice.com).

\section{B. Procedure}

In the first focus group study, physiotherapists were first given a demonstration of the Empatica device and an overview of PIS. This was followed by a discussion of their views on real-time monitoring and retrospective review of tracked data for individual self-management of $\mathrm{CP}$, for pain management programmes and during treatment sessions.

Sessions with people with $\mathrm{CP}$ were divided in three parts: a physical activity session (15 min), an overview and demonstration of PIS (10 min), and an interview (average length $42 \mathrm{~min}$ ). During the first session, each individual with $\mathrm{CP}$ did a bending exercise while wearing the device for data collection of BVP (heart rate, HRV, stress, relax), GSR (skin conductance, arousal, excitement), skin temperature (activity, context information), 3-axes accelerometer (movement, context information). Following this and similarly to the focus group, researchers gave participants an overview of PIS by following a script outlining the main characteristics and uses of PIS, and answered their questions; the Empatica mobile application was demonstrated and its capabilities explained (see Fig. 1-top right). Visualisation aspects of PIS were demonstrated through plotted graphs of biometric data collected by one of the researchers during an afternoon session (see Fig. 1bottom) together with visualisation of the data collected by participants during the physical exercise session, to show how monitoring could give insights into the relationship between pain-related physical/affective states and everyday activities.

Finally, a semi-structured interview was conducted to explore the use of PIS for pain self-management. Questions were framed around peoples' personal experiences, prompting them to think of challenging activities (e.g. repetitive tasks) and strategies they used (e.g. taking breaks). Participants were not constrained to talk about a particular device or monitoring method when responding.

Our studies had local Ethics Committee approval. All participants gave written consent. Focus groups and interviews were audio recorded and fully transcribed. Data was analysed using thematic analysis, to identify themes within a data set [31]. The transcripts were (re)coded iteratively following a coding framework developed by one coder and one reviewer. The web survey was posted for one month and people were contacted through twitter, charity groups such as BackCare, chronic pain websites and forums.

\section{FINDINGS}

Five main themes signifying possible roles of PIS for self-managing CP were identified: (i) assessment, planning 
and prevention (ii) a direct supervisory and co-management role, (iii) facilitating deeper understanding, (iv) managing emotional states, and (v) sharing for social acceptability. These themes are discussed below. Quotes for each theme are presented in Boxes 1-5 and identified in the text by an [ID]. Within each box, patients' quotes are indicated by (P\#) and physiotherapists' quotes by (PT\#).

\begin{tabular}{|l|l|}
\hline Box 1. Assessment, planning and prevention \\
\hline PA1 & $\begin{array}{l}\text { It's so much in life that you got to plan and do, and [pain] is an } \\
\text { added thing to me.[P6] }\end{array}$ \\
\hline PA2 & $\begin{array}{l}\text { [Clinicians] can see before a patient burns into flames, they can } \\
\text { see some smoke and they can do something about it. (P5). }\end{array}$ \\
\hline PA3 & $\begin{array}{l}\text { We have setbacks because we are over achievers. So it's about } \\
\text { having a setback plan ... ok, so my pain is increased, but what } \\
\text { emergency stuff do I need to do? Cut everything down by a bit? } \\
\text { Do I need to talk to the doctor? Do I need to increase my } \\
\text { medication?' (P5). }\end{array}$ \\
\hline $\begin{array}{l}\text { The ones who fill [questionnaires] in the waiting room five } \\
\text { minutes before they come in! So they're thinking 'what did I do } \\
\text { all week?'You know, how accurate is that recall. (PT1). }\end{array}$ \\
$\begin{array}{l}\text { This (PIS) gives us an idea of what (activity) you're doing for the } \\
\text { week, now let's work out how you could spread that out more } \\
\text { easily, or pace that a bit better. (PT1) }\end{array}$ \\
\hline
\end{tabular}

\section{A. Assessment, planning and prevention [Box1]}

Participants were interested in exploring sensing devices to support the planning and pacing of activities as this takes much of their day [PA1]. In this sense, physiotherapists confirmed that using PIS could helpfully oversee and prompt activity in patients, and incorporate multiple baselines for monitoring and evaluating outcomes of programmes [PA5]. PIS could further offer suggestions on areas patients could improve [PA5]. Also, sharing this data with healthcare professionals could help to facilitate a preventive approach for persistent illness care [PA2].

Previous research has primarily focused on providing medical data visualisations for practitioners [32], and there is a gap between information types needed to support medical treatment, and those that can help patients make sense of their own health-related experiences, related to their overall individual lifestyles, goals, and wellbeing [32]. For instance, patients may use collected bio-signal measures as a record of parameters they are requested to keep track of (e.g. mood diary, activity levels) rather than rely on recall when dealing with healthcare professionals [PA4]; this record can lead to a deeper and more objective understanding of pain management routines.

By looking at data tracked over longer periods people can get insights into what can be personalised or adapted; this is essential in CP where bad pain days and setbacks because of pain are a recurrent part of the condition. In addition, three of the participants expressed ideas for PIS to provide personalised advice and suggestions for what to do next [PA3]. Suggesting options for adapting activity and highlighting important points in the data to indicate meaningful aspects were considered useful functions.

\begin{tabular}{|l|l|}
\hline Box 2. Direct (real-time) supervisory and co-management role \\
\hline $\mathrm{DS} 1$ & $\begin{array}{l}\text { With my (golf) swing if my back feels stiff or tight, I'm waiting } \\
\text { for the moment for it to ping then I'm a bit slower than normal } \\
\text { in hitting it. I'm trying to concentrate on the golf, but not fully } \\
\text { because all the time I'm thinking about (pain). (P3). }\end{array}$ \\
\hline DS2 & $\begin{array}{l}\text { So when I get to a point, you'd get a bleep, or it comes up with } \\
\text { something that says - that's as far as you can go. If it was } \\
\text { connected to my spine and it would tell me how much } \\
\text { movement I have in my spine (P3) }\end{array}$ \\
\hline $\mathrm{DS3}$ & $\begin{array}{l}\text { If you had a glass body and could see some of the things that } \\
\text { are going on in there, you'd probably be able to do more (P3) }\end{array}$ \\
\hline
\end{tabular}

\begin{tabular}{|l|l|}
\hline Box 2 (continued) Concerns \\
\hline DS4 & $\begin{array}{l}\text { It's not that we want them to be focused on their pain all the } \\
\text { time, but they have to be actively planning and managing it } \\
\text { and, to an extent, this (PIS) is removing that. (PT1). }\end{array}$ \\
\hline DS5 & $\begin{array}{l}\text { I worry it will make them more vigilant about the stress and } \\
\text { we're trying to make them less vigilant." (PT3). }\end{array}$ \\
\hline DS6 & $\begin{array}{l}\text { I'm a bit concerned that some of our patients would not want } \\
\text { any stress at all, and they might end up doing very little. (PT2) }\end{array}$ \\
\hline
\end{tabular}

\section{B. Direct (real-time) supervisory and co-management role) [Box 2]}

Other than planning, monitoring pain while doing an activity can have a disruptive effect on the ability to perform it 'normally' [DS1]. Participants expressed a need to transfer some of the responsibility for monitoring their activity and pain to the technology, freeing themselves from having to continually plan and pace activities. Participants wanted to be warned when they were going too far or doing too much by tracking physical parameters in real-time [DS2]. This information would also give them options for adapting activity in real-time. However, physiotherapists were concerned that this may lead people to adopt a passive stance to managing their pain and becoming focused on more negative aspects such as stress [DS4, DS5], which they thought may be interpreted as 'right or wrong' responses [DS6] rather than as broad representations of emotion [31]. In contrast to the physiotherapists' view, people with CP visualised the device as a means to see inside the body to better infer one's physical and emotional state from feedback and take more informed actions [DS3].

\begin{tabular}{|l|l|}
\hline $\begin{array}{l}\text { Box 3. Facilitating deeper understanding (a pain management } \\
\text { buddy) }\end{array}$ \\
\hline PB1 & $\begin{array}{l}\text { For my condition and on-going wellbeing I need to stay moving, } \\
\text { because in 20 years' time, how uncomfortable is this condition } \\
\text { going to be? And (PIS) could be quite useful. (P8) }\end{array}$ \\
\hline PB2 & $\begin{array}{l}\text { I have a deterioration of the facet joints and I don't know what's } \\
\text { happening. I'm off the medication and I get pain there, but it's } \\
\text { not as bad as it used to be. But I don't know why - Do I manage } \\
\text { it better? Has it healed itself? (P3). }\end{array}$ \\
\hline PB3 & $\begin{array}{l}\text { When a patient sees the doctor or healthcare professional [...] } \\
\text { they know they got pain. But the biggest problem is sleep, or they } \\
\text { are stressed out, or they are gaining weight, or they got } \\
\text { depression [...], all this stuff supersedes (pain). (P5). }\end{array}$ \\
\hline PB4 & $\begin{array}{l}\text { It will be interesting to know [how stiff my muscles are]. Part of } \\
\text { [my] treatment is measuring ability to stretch and move. If I } \\
\text { could [measure stiffness] on a more regular basis that would be } \\
\text { really useful. (P8). } \\
\text { Ialways ask myself, 'Is the type of shoes I'm wearing what makes } \\
\text { me feel (pain)?' (P7). }\end{array}$ \\
\hline
\end{tabular}

\section{Facilitating deeper understanding (a pain management buddy) [Box 3$]$}

One of the core stages of PIS involves considering motivations for self-tracking [1]. In this sense, capturing personal information for long-term conditions can assist people with $\mathrm{CP}$ to better manage pain, rather than find a cure [21]. At least 2 of the participants were concerned about the long-term trajectory of their pain as they got older and this was their motivation for long-term monitoring [PB1]. Participants also wanted to gain a better understanding of the $\mathrm{CP}$ condition not provided by current medical knowledge [PB2]. Many participants also expressed interest in establishing correlations of pain with lifestyle aspects that can have an impact on their wellbeing such as sleep [PB3], hydration levels, or stiffness [PB4]. 


\begin{tabular}{|c|c|}
\hline \multicolumn{2}{|c|}{ Box 4. Managing Emotional States } \\
\hline ME1 & $\begin{array}{l}\text { I might be stressed, but I'd like to check to make sure I'm not } \\
\text { being overly emotional about it, the device could work for } \\
\text { that.(P1). } \\
\text { If you can see it, rather than just feeling it 'oh, I must need to de- } \\
\text { stress!'[...] help get your stress levels down, be aware. (P9). }\end{array}$ \\
\hline ME2 & $\begin{array}{l}\text { Something with physical contact where I'd notice a vibration [to } \\
\text { check] what's going on, and it would trigger that I was getting } \\
\text { to threshold, something that would help control my day. (P4). }\end{array}$ \\
\hline ME3 & $\begin{array}{l}\text { Stress levels will increase my levels of anxiety and if I could } \\
\text { monitor that then that would be very useful, because anxiety } \\
\text { triggers virtual paranoia and then pain, } a \text { wicked circle (P4). }\end{array}$ \\
\hline ME4 & $\begin{array}{l}\text { It might help me to see [...] where triggers are happening. (P5) } \\
\text { Sometimes you go through quite harsh pain, and you think where } \\
\text { did that come from? if that was recorded, how the body was } \\
\text { reacting, and you can go back and forth with that to find 'that } \\
\text { was that time!' So you could avoid it another time. (P7). }\end{array}$ \\
\hline ME5 & $\begin{array}{l}\text { I read a story once about a guy that walked up a steep hill and } \\
\text { looked up and said 'oh my god, look how far I got to go!' But } \\
\text { then, he turned round to see how far he'd come. So sometimes, } \\
\text { we need information, how much we have progressed. (P5). }\end{array}$ \\
\hline \multicolumn{2}{|c|}{ Concerns } \\
\hline ME6 & $\begin{array}{l}\text { If they do too many diaries they may get hypervigilant, and } \\
\text { worry 'the app says do that, stop now, don't do that', they start } \\
\text { to think 'that must be bad for me'. (PT2). }\end{array}$ \\
\hline ME7 & $\begin{array}{l}\text { "So if it's really hot outside and you're sweating, it won't } \\
\text { necessarily assume that you are stressed?" (PT2). }\end{array}$ \\
\hline
\end{tabular}

\section{Managing emotional states [Box 4]}

PIS emerged as an objective means to validate painrelated affective experiences in real-time, such as recognising when a relatively safe activity triggers disproportionate amounts of anxiety [ME1]. Real-time feedback of biometric measures could enhance the sense of control [ME2] when approaching challenging activities, for example, by providing users with routes of action to revert to baseline levels [ME3] such as a predefined 'normal heart rate'. A majority of participants also expressed interest in better understanding their pain and its associated emotional states and patterns of behaviour in the short/medium term so they could gain insights to act upon [ME4]. Visualising data in retrospect can also help to highlight pain-related data facets such as when progress is slow [12]. However, a balance is necessary between reporting long-term progress (which may be slow) and more regular feedback to ensure motivation in maintenance of function [ME5]. In light of this, physiotherapists felt technology should be prescribed on a 'right patient, right time basis' since some patients could be more prone to wrongly interpret bio signals [ME6]. They also expressed concern about the accuracy of devices, e.g., their ability to distinguish between biomarkers from body signals and external factors such as heat [ME7].

\begin{tabular}{|l|l|}
\hline \multicolumn{2}{|c|}{ Box. 5 Sharing for social acceptability } \\
\hline SA1 & $\begin{array}{l}\text { Other people don't always know how I'm feeling (pain) so I've } \\
\text { learned to communicate with people a lot better [...] I just } \\
\text { think whether we can create one of these (baby on board } \\
\text { badges) for a disabled person. (P2) }\end{array}$ \\
\hline SA2 & $\begin{array}{l}\text { I do not have a good relationship with my GP at all, so it was } \\
\text { more trying to track these other things that are going on rather } \\
\text { than saying I know you don't believe me (P2) }\end{array}$ \\
\hline SA3 & $\begin{array}{l}\text { It could just be one thing that will trigger [the pain] to such a } \\
\text { degree that I am on heightened state, I'm not comfortable with } \\
\text { people, I'm using harsher language, [...]. (P5) }\end{array}$ \\
\hline SA4 & $\begin{array}{l}\text { It would help control my day and not be controlled by } \\
\text { others. When you feel you are in charge, you feel better. (P2) }\end{array}$ \\
\hline
\end{tabular}

\section{E. Sharing for social acceptability [Box 5]}

$\mathrm{CP}$ is an invisible condition and the distress caused by it may not be obvious to even close ones [SA1]. Some participants felt that their condition is disbelieved or minimised, even by healthcare professionals who they turn to for help [SA2]. In these cases, PIS could be used to gather and provide physical evidence of pain, so others would be more understanding and supportive. This desire for trust and support also extended to general others (in addition to family, friends and healthcare providers) to address situations such as having to ask for a seat on a train when pain increased. Participants felt that PIS could also help them to function better in social situations by nudging them if they were getting stressed [SA3]. They saw this as a tool to regain control [SA4].

\section{F. What to track: survey results}

Most people who completed the survey reported pain in multiple body parts (Fig. 2a). Only 7 of them were tracking information, in accordance with the literature showing limited adherence to manual tracking [21]. Only three of them shared information with a $\mathrm{GP} /$ physiotherapist (e.g., one person used dropbox to share data with the clinic). They reported tracking pain levels, activities, food intake, sleeping patterns and weather using smartphone apps, pedometers, paper diaries, computer applications and smartwatches.

When asked what they would like to track using emerging sensing technology, movement/posture, muscle activity and anxiety came at the top of the list but just slightly above other physiological information (Fig. 2b). Mood received less interest. People felt that both kinematic and physiological information could be useful for managing and pacing activity; tracking anxiety was considered important as well but less so than kinematic and physiological information. People reported that they wanted to see the data in real-time (Fig. 2c). However a record of tracked data for reflection was also considered useful.

Out of 35 respondents, 21 expressed interest in sharing some of the data with their GP/ physiotherapist (Fig 2d) but they were less ready to share information with others as it was considered private. 18 participants would share data with a partner, 7 with friends, 4 with colleagues and only 3 with unknown others. People felt pain level was most useful to share with friends (Fig 2d), possibly to feel better understood and supported. Sharing some data in a public space was considered useful in case the person required help and it was suggested that the device should be able to call for help. Participants also felt that such data could help others understand them better and facilitate social inclusion.

\section{DISCUSSION}

Five possible roles of PIS technology for CP emerged from the study. Three roles overlap with those suggested by PIS literature for healthy people (e.g., directive tracking, documentary tracking and diagnostic tracking [33]). These relate to the need that emerged here for a better understanding of one's behaviour, one's condition and its management. Our survey study also showed that half of the people that are already tracking data, shared their data with health providers to improve communication and treatment. This sharing of tracked data has been seen in other clinical applications to evaluate effects of therapy [41] or facilitate coping with threatening episodes (e.g., bipolar disorder [23]). Beyond these similar roles found in the literature, our study identified two PIS roles more specific to CP: (i) allowing the diversion of cognitive resources from pain attention and planning to improving function; (ii) gathering evidence for oneself and for others, important because CP is an invisible condition. Here, we discuss these two specific aspects, only touching upon the other roles when needed. 


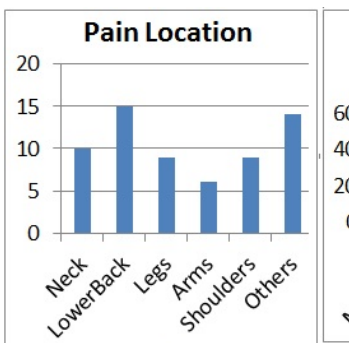

(a)

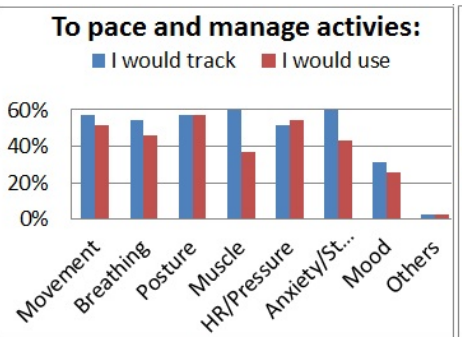

(b)

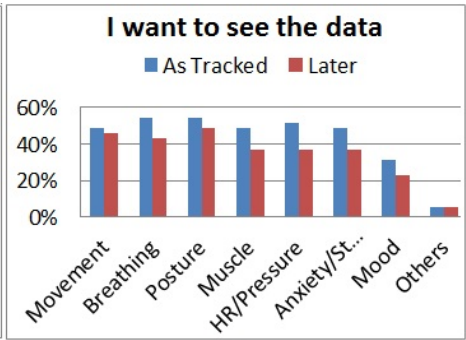

(c)
I would share this information with:

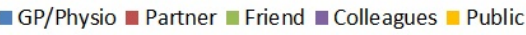
$60 \%$

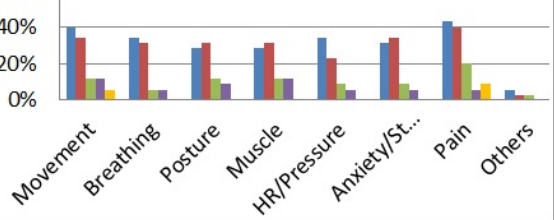

(d)

Fig. 2. Results from the web-based surveys on the use of tracking technology

\section{A. A shared supervisory role to redirect cognitive resources}

Singh et al. discuss the use of sensing technology for personalising physical activity sessions to make them more effective and reduce focus on pain [9]. What emerged from our study is that personalisation is also important to facilitate everyday functioning since planning and monitoring tasks occupy much of the daily life of people with CP [9] and changes to routine are challenging. Often, people reduce social participation or non-essential activities outside the home to avoid unfamiliar situations predicted to exacerbate pain [7]. Additionally, altering a plan or routine may have critical costs such as increased pain [4] and not being able to accomplish important tasks (e.g., picking up child from school). Having a device that helps to not just plan daily activities but to adapt the plan in real-time according to available resources could provide valuable support. For example, by suggesting sitting if muscle tension is detected during prolonged standing in social settings, by providing help with pacing a walk (e.g., alternate route suggestions in case of unexpected events), or by prompting better breathing if breath becomes shallow.

For this, the PIS device must access the information necessary for estimating psychological and physical resources in real-time, according to activities already performed, the current activity, and forthcoming demands. Most sensing devices focus on measuring heart rate, GSR and gross behaviour but our qualitative study and the survey suggest the usefulness of additional sensing capabilities such as measuring muscle activity and tracking more specific body movements (especially those that induce pain). Beyond tracking and pacing the amount of activity performed by any part of the body, sensing technology could help to identify early signs of tension and use of protective behaviour that could be predictors of increased pain and associated misconceptions [15]. Recent results from the affective computing literature [34], [35] show the possibility to automatically detect perceived pain level and protective behaviour due to anxiety during CP physical rehabilitation by using full motion capture technology and electromyography sensors. The challenges are to transfer these functions to minimal wearable sensing devices and to identify ways to gather contextual information, such as what activity is being performed (e.g., sit-to-stand, bending to load the washing machine), to facilitate the detection of how it is performed (i.e., use of protective strategies when increased pain or anxiety). Progress in this direction is being made as PIS get better at differentiating types of physical activity [27]. In addition, position data from GPS and video (e.g., e-glasses [36]), may contribute to building such contexts to better understand for example if the cause of protective behaviour is anxiety or environmentally related (e.g., crossing a wide road vs space in front of the chair while standing up).

Beyond the critical issues of availability and reliability of these measures while on the move and the importance of the situated aspect of wearable PIS technology, it is worth considering to what extent the device should adopt a supervisory role. Physiotherapists raised two important concerns: first, by transferring the responsibility for monitoring and planning to a device, the person with $\mathrm{CP}$ may become dependent on it and not develop the skills to manage his/her own condition [9]. This can be addressed by using the device as a lens to facilitate reading one's body and needs without dictating responses or their timing. Self-directed calibration and feedback techniques proposed for specific training sessions $[9,37]$ could be translated to fit the broader context of functioning and to set the degree of supervision. For example, in very cognitively demanding contexts (e.g., a work meeting), more tracking aspects could be used to alert when action is needed (e.g., changing postures, stretching), while in less cognitively demanding contexts, the device could mainly be used to feed information to the user in the background.

A second concern for physiotherapists was that users could rely on the device for cues of risk of pain increase or injury. The resultant increase in anxiety and focus on pain could lead to further avoidance of activity wrongly construed as risky to the detriment of their quality of life. Results from [9], [38] show that pleasurable representation of tracked information (movement) enabled people to concentrate on important aspects of movement and shifted their focus from pain. This is possibly the case even beyond physical activity sessions. The challenge is to provide an informative but easily attended pleasurable representation. In addition, PIS could tailor information differently when detecting negative reactions to motivate and adjust activities rather than avoid them.

\section{$B$. Gathering evidence for oneself and for the others}

Beyond sharing information with health providers and partners for better care, our study highlighted the opportunity to use PIS to gather evidence of one's invisible health condition. Such physical evidence of a subjective complex experience could help them to achieve a better sense of control over their own life as in [38] and lead to more social inclusion because others have a better understanding of their condition.

Evidence for self: Participants in our study expressed a desire to understand behaviours associated with their CP so 
they could cope better as they age, or because current medical knowledge cannot fulfil their need for understanding. This is congruent with the concept of illness perception [39] in information visualisation for health technologies, which posits that the ways in which a person deals with their condition are associated with their beliefs about their illness. PIS offers the possibility of increased understanding of the condition and can help to self-correct unhelpful beliefs, such as that pain equals harm, particularly during a crisis. As acute physical pain is an evolutionary and beneficial response to any damage to one's body or the possibility thereof, it is difficult to accept that this is not the case in CP. Also, differentiating CP from normal pain (e.g., due to overuse of joints or muscles) is important [11] for reducing stress and better activity pacing. Our survey showed that kinematic and physiological information was important to participants to facilitate direct action based on the data as well as post-reflection on the data to facilitate selfmanagement.

Evidence for others: Information collected through PIS could help others to better understand the $\mathrm{CP}$ condition, given that many people with CP face social isolation. For example, participants mentioned wanting a transparent body that could be read and understood by themselves and others to foster acceptance and overcome stigma. The literature shows that moral judgements are made about people exhibiting pain behaviour when physical evidence of impairment is absent [40][41]. Instead, use of PIS can facilitate communication of difficulties and needs. The question for the PIS designer then is what aspect of the embodied CP experience could be disclosed to communicate that $\mathrm{CP}$ is real while still ensuring privacy. Our survey shows that different levels of transparency are needed depending on social context (e.g., family vs work, colleagues vs strangers), level of interaction and the needs fulfilled by social context. In particular, people considered kinematics and physiological measurements data important for themselves and carers but wanted to restrict sharing to mostly pain level information with non-carer others. While sharing information with others can facilitate understanding and support, the risk is of over-empathic behaviour or sympathy that is not always what people with $\mathrm{CP}$ need or want [42] as it can feel like pity and lack of respect.

\section{Towards an extended model for PIS}

From the above discussion, there is clearly a need to expand the previously proposed models of PIS. Firstly, current PIS models propose a linear configuration of the stages of collecting, reflecting and taking action on collected data [1]. These models mainly focus on facilitating reflection and better understanding of one's condition to enable behaviour change or improve wellbeing. Our study suggests a more complex pattern where PIS may also be used to act on data in realtime, with the possibility to reflect on it afterwards. For example, unjustified prolonged increases in muscle activity may require immediate action to reduce the chance of increased pain. Also, an immediate reaction may be followed by cycles of reflection-action to facilitate the activity being accomplished. This non-linear process also suggests that data processing and feedback may differ according to how it will be used. Factors such as the ability of the person to cope with their condition may also mediate such representations.
Secondly, multiple consumers of this tracked data may exist. Beyond sharing data with healthcare providers for more effective care, our study highlights the needs for sharing aspects of the tracked data with social others to gain trust, understanding and social acceptance, a PIS social role largely ignored. Differently from MacLeod et al. [21] that reflect on the social embarrassment of collecting data, our participants highlighted instead its social opportunities. However, the pain experience is private. Hence, designing for such social sharing may require specific data processing, representation and different levels of access from those required for the self or the carer. In addition, there is a need to understand what evidence of $\mathrm{CP}$ would be trusted by others, especially more distant others (co-workers or unknown people in public spaces). Inferring pain levels from non-verbal behaviour [35] can be a useful way to personalize feedback for the self. However, others may feel that this behaviour is faked as shown in [40]. In this case, physiological and neural-based evidence (e.g., detecting pain from EEG ([43]) could be effective solutions since people have lesser control on these, but privacy is still a concern with this data.

\section{CONCLUSION}

The results from our studies with physiotherapists and people with CP emphasise that tracking data such as kinematic and physiological data as well as anxiety can be useful to better manage CP. Among roles for PIS that emerged, two were specific to $\mathrm{CP}$ : (i) redirecting cognitive resources and (ii) gathering evidence for oneself and others. In the discussion section, we highlighted how physiotherapists concerns about PIS undermining self-management could be addressed by: sharing responsibility rather than fully transferring supervision and calibration functions, providing pleasurable representations of relevant information that are easy to attend to and use without increasing attention to pain and stress. Additionally, while pain is a private experience, people with CP want to share necessary and pertinent information. All these results show that PIS models should be extended to include real-time use of data in addition to that available later for reflection. Additionally, there is a need for multiple but controlled data sharing for social purposes, not just for better care. These needs for sharing call for further studies to better understand the perspective of the people with CP in terms of what they want to share, what is useful for other consumers of this data to receive, as well as the barriers, needs, benefits and implications that may arise from sharing such information.

\section{ACKNOWLEDGMENTS}

Thanks to EPSRC EP/G043507/1 grant: Pain rehabilitation: E/Motion-based automated coaching (www.emo-pain.ac.uk). C. Bradley was supported by the EPSRC EP/G004560/1 UCLIC/FIT Lab Platform grant. Thanks to physiotherapists at the University College London Hospital for their participation.

\section{REFERENCES}

[1] I. Li, A. Dey, and J. Forlizzi, “A stage-based model of personal informatics systems," Proc. 28th Int. Conf. Hum. factors Comput. Syst. CHI '10, p. 557, 2010.

[2] E. Thomaz, "A Human-centered conceptual model for personal health informatics data," in Pers. Inform. in the Wild: Hacking Habits for Health \& Happiness - CHI 2013 Workshop, 2013. 
[3] M. S. McDermott and A. E. While, "Maximizing the healthcare environment: a systematic review exploring the potential of computer technology to promote self-management of chronic illness in healthcare settings," Patient Educ. Couns., Apr. 2013.

[4] R. J. Gatchel, Y. B. Peng, M. L. Peters, P. N. Fuchs, and D. C. Turk, "The biopsychosocial approach to chronic pain: scientific advances and future directions," Psychol. Bull., vol. 133, no. 4, pp. 581-624, 2007.

[5] G. Crombez, C. Eccleston, S. Van Damme, J. W. S. Vlaeyen, and P. Karoly, "Fear-avoidance model of chronic pain," Pain,vol. 28, no. 6, pp. 475-483, 2012

[6] I. Tracey and M. C. Bushnell, "How neuroimaging studies have challenged us to rethink: is chronic pain a disease?," J. Pain, vol. 10, no. 11. pp. 1113-1120, 2009.

[7] A. Benjamin, J. Birnholtz, R. Baecker, D. Gromala, and A. Furlan, "Impression management work," in Proc. of ACM 2012 Conf. Computer Supported Cooperative Work - CSCW '12, 2012, p. 799.

[8] M. O. Martel, P. Thibault, and M. J. L. Sullivan, "Judgments about pain intensity and pain genuineness: the role of pain behavior and judgmental heuristics," J. Pain, vol. 12, no. 4, pp. 468-475, 2011.

[9] A. Singh, A. Klapper, J. Jia, A. Fidalgo, A. Tajadura-Jiménez, N. Kanakam, N. Bianchi-Berthouze, and A. Williams, "Motivating people with chronic pain to do physical activity: opportunities for technology design," in CHI'14, 2014, pp. 2803-2812,

[10] C. Harstall and M. Ospina, "How prevalent is chronic pain?," Pain Clin. Updat., vol. XI, no. 2, pp. 7-9, 2003.

[11]M. Geisser, M. Robinson, F. Keefe, and M. Weiner, "Catastrophizing, depression and the sensory, affective and evaluative aspects of chronic pain," Pain, vol. 59, pp. 79-83, 1994.

[12] M. P. Jensen, J. a Turner, and J. M. Romano, "Changes after multidisciplinary pain treatment in patient pain beliefs and coping are associated with concurrent changes in patient functioning," Pain, vol. 131, no. 1-2, pp. 38-47, Sep. 2007.

[13] L. Ruehlman, P. Karoly, and C. Enders, "A randomized controlled evaluation of an online chronic pain self management program," Pain, vol. 153, no. 2, pp. 319-330, 2012.

[14] M. J. L. Sullivan and W. D. Stanish, "Psychologically based occupational rehabilitation: the pain-disability prevention program.," Clin. J. Pain, vol. 19, no. 2, pp. 97-104, 2003.

[15] V. Harding and A. Williams, "Extending physiotherapy skills using a psychological approach: cognitive-behavioural management of chronic pain," Physiotherapy, vol. 81, no. 11, pp. 681-688, Nov. 1995.

[16] J. L. Bender, A. Radhakrishnan, C. Diorio, M. Englesakis, and A. R. Jadad, "Can pain be managed through the internet? a systematic review of randomized controlled trials.," Pain, vol. 152, no. 8, pp. 1740-50, 2011.

[17] O. B. Kristjánsdóttir, E. a Fors, E. Eide, A. Finset, T. L. Stensrud, S. van Dulmen, S. H. Wigers, and H. Eide, "A smartphone-based intervention with diaries and therapist-feedback to reduce catastrophizing and increase functioning in women with chronic widespread pain: randomized controlled trial," J. Med. Internet Res., vol. 15, no. 1. p. e5, Jan-2013.

[18]C. Rini, D. Williams, J. Broderick, and F. Keefe, "Meeting them where they are: using the internet to deliver behavioral medicine interventions for pain,” Behav. Med., vol. 2, no. 1, pp. 82-92, 2012.

[19] Y. Huang, H. Zheng, C. Nugent, P. McCullagh, N. Black, K. E. Vowles, and L. McCracken, "Feature selection and classification in supporting report-based self-management for people with chronic pain," IEEE Trans. Inf. Technol. Biomed., vol. 15, no. 1, pp. 54-61, Jan. 2011.

[20] N. Pombo, P. Araújo, and J. Viana, "Contribution of Web Services to Improve Pain Diaries Experience,” in Proc. Int. MultiConf. Eng. Comp. Scient., vol. I, pp. 14-17, 2012.

[21] H. MacLeod, A. Tang, and S. Carpendale, "Personal Informatics in Chronic Illness Management,” in Graph. Interf. 2013, pp. 149-156.

[22]F. Spyridonis, J. Hansen, T.-M. Grønli, and G. Ghinea, "PainDroid: an android-based virtual reality application for pain assessment," Multimed. Tools Appl., Jan. 2013.

[23] J. E. Bardram, M. Frost, K. Szántó, M. Faurholt-Jepsen, M. Vinberg, and L. V. Kessing, "Designing mobile health technology for bipolar disorder: a field trial of the monarca system," in Proc. SIGCHI Conf. on Human Factors in Comp. Systems, 2013, pp. 2627-2636.

[24]B. A. Rosser, K. E. Vowles, E. Keogh, C. Eccleston, and G. A. Mountain, "Technologically-assisted behaviour change: a systematic review of studies of novel technologies for the management of chronic illness," J. Telemed. Telecare, vol. 15, no. 7, pp. 327-38, Jan. 2009.

[25]C. Perera, "The evolution of e-health - mobile technology and mhealth," J. Mob. Technol. Med., vol. 1, no. 1, pp. 1-2, Mar. 2012.

[26] S. Patel, H. Park, P. Bonato, L. Chan, and M. Rodgers, "A review of wearable sensors and systems with application in rehabilitation," J. Neuroeng. Rehabil., vol. 9, no. 1, p. 21, Jan. 2012.

[27]D. Morris, T. Saponas, A. Guillory, and I. Kelner, "Recofit: using a wearable sensor to find, recognize, and count repetitive exercises," in CHI'14 Proc. SIGCHI Conf. on HF in Comp. Sys., 2014, pp. 3225-3234.

[28]F. Casamassima, A. Ferrari, B. Milosevic, L. Rocchi, and E. Farella, "Wearable audio-feedback system for gait rehabilitation in subjects with Parkinson's disease," in Proc. 2013 ACM Conf. Pervas. and Ubiq. Comp. adjunct publication, 2013, pp. 275-278.

[29] C. Schonauer and T. Pintaric, "Chronic pain rehabilitation with a serious game using multimodal input," Virtual Rehabil., 2011.

[30]D. Gromala, M. Song, J.-D. Yim, T. Fox, S. J. Barnes, M. Nazemi, C. Shaw, and P. Squire, "immersive vr : a non-pharmacological analgesic for chronic pain ?,” in Proc. Annual Conf. on HF in Comp. Sys. (CHI '11), 2011, pp. 1171-1176.

[31]H. Joffe and L. Yardley, "Content and thematic analysis," in Research Methods for Clinical and Health Psychology, D. F. Marks and L. Yardley, Eds. Sage Publications Ltd, 2003, pp. 56-68.

[32] C. Fan, "The Future of data visualization in personal informatics tools," in Personal Inform. in the Wild: Hacking Habits for Health \& Happiness CHI 2013 Workshop, 2013

[33] J. Rooksby, M. Rost, A. Morrison, and M. C. Chalmers, "personal tracking as lived informatics," in Proc. 32nd Annual ACM Conf. on HF in Comp. Sys., 2014, pp. 1163-1172.

[34] M. Aung, N. Bianchi-Berthouze, P. Watson, and A. C. de C. Williams, "Automatic Recognition of fear-avoidance behavior in chronic pain physical rehabilitation," in Pervas. Comp. Techn. Healthc., 2014, pp. 0-3.

[35] T. Olugbade, M. Aung, N. Marquardt, N. Bianchi-Berthouze, and A. de C Williams, "Bi-modal detection of painful reaching for chronic pain rehabilitation systems," Sep. 2014.

[36] J. Hernandez and R. W. Picard, "SenseGlass," in Proc. of the adjunct publication of 27th annual ACM symposium on User interface software and technology - UIST'14 Adjunct, 2014, pp. 77-78.

[37] R. Johnson, N. Bianchi-Berthouze, Y. Rogers, and J. van der Linden, "Embracing calibration in body sensing: using self-tweaking to enhance ownership and performance," UbiComp '13 Proc. 2013 ACM Int. Jt. Conf. Pervasive Ubiquit. Comput., p. 811, 2013.

[38] A.Singh et al. "Go with the flow: Tracking, analysis and sonification of movement and breathing to build confidence in activity despite chronic pain," Human-Computer Interaction (in revision).

[39]S. Faisal, A. Blandford, and H. W. Potts, "Making sense of personal health information: challenges for information visualization," Health Informatics J., vol. 19, no. 3, pp. 198-217, Sep. 2013.

[40]C. E. Ashton-James, D. C. Richardson, A. C. de C Williams, N. BianchiBerthouze, and P. H. Dekker, "Impact of pain behaviors on evaluations of warmth and competence," Pain, vol. 155, no. 12, pp. 2656-61, Dec. 2014.

[41] M. S. H. Aung, et al., "The automatic detection of chronic pain-related expression: requirements, challenges and a multimodal dataset," Trans. Affective Computing, 2015

[42] J. Rainville, R. J. E. M. Smeets, T. Bendix, T. H. Tveito, S. Poiraudeau, and A. J. Indahl, "Fear-avoidance beliefs and pain avoidance in low back pain-translating research into clinical practice.," Spine J., vol. 11, no. 9, pp. 895-903, Sep. 2011.

[43] S. Schmidt, J. R. Naranjo, C. Brenneisen, J. Gundlach, C. Schultz, H. Kaube, T. Hinterberger, and D. Jeanmonod, "Pain ratings, psychological functioning and quantitative EEG in a controlled study of chronic back pain patients.," PLoS One, vol. 7, no. 3, p. e31138, Jan. 2012. 